\title{
BMJ Open Training frequency for educating schoolchildren in basic life support: very brief 4-month rolling-refreshers versus annual retraining-a 2-year prospective longitudinal trial
}

Cristian Abelairas-Gómez (D) , 1,2,3 Santiago Martinez-Isasi (iD) ,1,3,4

Roberto Barcala-Furelos, ${ }^{5,6}$ Cristina Varela-Casal, ${ }^{5,6}$ Aida Carballo-Fazanes (D) , 1,3,4 María Pichel-López, ${ }^{5}$ Felipe Fernández Méndez, ${ }^{5}$ Martín Otero-Agra, ${ }^{5}$ Luis Sanchez Santos, ${ }^{7}$ Antonio Rodriguez-Nuñez ${ }^{1,3,4,8}$

To cite: Abelairas-Gómez C, Martinez-Isasi S, BarcalaFurelos R, et al. Training frequency for educating schoolchildren in basic life support: very brief 4-month rolling-refreshers versus annual retraining-a 2-year prospective Iongitudinal trial. BMJ Open 2021;11:e052478. doi:10.1136/ bmjopen-2021-052478

- Prepublication history for this paper is available online. To view these files, please visit the journal online (http://dx.doi. org/10.1136/bmjopen-2021 052478).

Received 04 May 2021 Accepted 25 October 2021

Check for updates

(C) Author(s) (or their employer(s)) 2021. Re-use permitted under CC BY-NC. No commercial re-use. See rights and permissions. Published by BMJ.

For numbered affiliations see end of article.

Correspondence to Dr. Santiago Martinez-Isasi; smtzisasi@gmail.com

\section{ABSTRACT}

Objective To compare the effectiveness of 4-month rollingrefreshers and annual retraining in basic life support (BLS) on a sample of schoolchildren.

Design Prospective longitudinal trial.

Setting and participants Four hundred and seventy-two schoolchildren (8-12 years old).

Interventions Schoolchildren were instructed in BLS and then split into the following three groups: control group (CG), standard group (SG) and rolling-refresher group (RRG). Their BLS skills were assessed within 1 week (T1) and 2 years later (T2). Moreover, CG did not receive any additional training; $S G$ received one 50 min retraining session 1 year later; $R R G$ participated in very brief ( $5 \mathrm{~min}$ ) rolling-refreshers that were carried out every 4 months.

Primary and secondary outcomes Hands-on skills of BLS sequence and cardiopulmonary resuscitation.

Results BLS sequence performance was similar in all groups at T1, but SG and RRG followed the steps of the protocol in more proportion than CG at T2. When compared at T2, RRG showed higher proficiency than SG in checking safety, checking response, opening the airway and alerting emergency medical services. In addition, although the mean resuscitation quality was low in all groups, RRG participants reached a higher percentage of global quality cardiopulmonary resuscitation (CG: 16.4 24.1 ; SG: 25.3 \pm 28.8 ; RRG:

$29.9 \% \pm 29.4 \%$ ), with a higher percentage of correct chest compressions by depth (CG: $3.9 \pm 11.8$; SG: $10.8 \pm 22.7$; RRG: $15.5 \pm 26.1 \mathrm{~mm}$ ).

Conclusions In 8-to-12-year-old schoolchildren, although annual 50 min retraining sessions help to maintain BLS performance, 4-month very brief rolling-refreshers were shown to be even more effective. Thus, we recommend implementing baseline BLS training at schools, with subsequently brief rolling-refreshers.

\section{INTRODUCTION}

Basic life support (BLS) education of schoolchildren, combined with other community strategies, has been shown to be an effective

\section{Strengths and limitations of this study}

- The recruited schools were not randomly selected, although the allocation of the groups was randomised. In addition, schoolteachers were not observed while teaching, so consistency between schoolteachers is unknown.

- The simulated scenario presented to participants, in which psychological variables typical of real scenarios are not present, makes it necessary to interpret the results with caution.

- As usual in training activities, manikins were used to teach and test basic life support.

- The results were not disaggregated by age, which might show some differences in the effect of rollingrefreshers depending on the age of schoolchildren. However, anthropometric variables (weight, height and body mass index) were registered and analysed since it was shown that the quality of cardiopulmonary resuscitation performed by schoolchildren depends on these variables.

measure to increase witness-assisted out-ofhospital cardiac arrest. ${ }^{1}$ This is the main reason why both first aid and BLS are promoted to be included in school curricula, ${ }^{2-4}$ which is the gold goal of the KIDS SAVE LIVES statement

Previous studies have already shown the capacity of schoolchildren to learn BLS sequences, ${ }^{5-7}$ cardiopulmonary resuscitation $(\mathrm{CPR})^{6-8}$ and automated external defibrillation use. ${ }^{79}$ Therefore, it is proven that they are able to learn. However, similar to adult laypeople, schoolchildren's BLS education and knowledge retention cannot depend only on one-off trainings. In this sense, the inclusion of BLS in school curricula should imply that schoolchildren train periodically. ${ }^{3410}$ 
The KIDS SAVE LIVES statement recommends 2 hours of CPR training up to the age of 12 years, although it could start earlier. ${ }^{11}$ An evidence-based educational pathway was also designed to help schoolteachers and academic institutions integrate BLS training into school curricula, ${ }^{2}$ and different education tools were designed and validated to educate and/or assess BLS schoolchildren's knowledge/skills. ${ }^{12}{ }^{13}$ However, to the best of our knowledge, no longitudinal trials have been carried out to study different BLS training approaches in school environments in terms of methodology. We are aware of only one longitudinal (more than 1 year) study that compared groups of schoolchildren trained by schoolteachers or emergency physicians to determine whether teaching staff were as able as medical staff to teach BLS. ${ }^{14}$ This may be because it poses a challenge in terms of teaching methodology, educators (schoolteachers or health staff) and resources (human and material).

Limited retention of BLS knowledge/skills over time is one of the barriers to high-quality CPR, which is a reason to design and test new methodologies to efficiently teach and maintain these competencies. 'Rolling-refreshers', consisting of periodically repeated brief hands-on sessions, is a novel approach to maintain CPR psychomotor skills, initially tested and recommended for healthcare personnel. ${ }^{1516}$ Nonetheless, it was also shown to be effective in teaching and retaining other abilities as a BLS sequence. ${ }^{17}$

We hypothesised that the rolling-refreshers approach is more effective in maintaining schoolchildren's BLS skills over time than annual retraining. Therefore, the objective of the present study was to compare every 4-month 5 min rolling-refreshers with $50 \mathrm{~min}$ annual retraining in a sample of 8-to-12-year-old schoolchildren.

\section{METHODS}

\section{Participants}

A convenience sample of 658 schoolchildren was invited to participate. The participants were from three different city-based schools, which comprised the three cohorts of the study (see Design section). Schoolchildren were 8-12 years old, which corresponded with Spain's third to sixth grades of elementary education.

The objectives and methods of the study were explained to parents/guardians, who authorised the participation of schoolchildren by signing an informed consent form. This also meant that they understood the voluntary character of the participation and that they could withdraw at any moment. In addition, verbal assent from each child was required. The Ethical Committee of the Faculty of Education and Sport Sciences-University of Vigo (Spain)—approved the study protocol. Participants with any physical or psychological impairment were excluded, as well as those whose parents or legal guards denied consent to participate. Schoolchildren who did not attend both BLS assessments were also excluded.

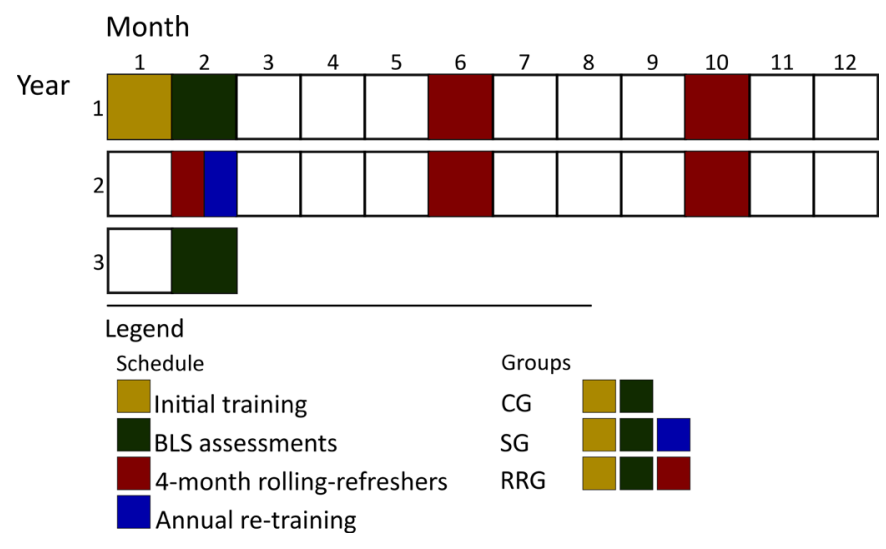

Figure 1 Timetable of the longitudinal design.

Patient and public involvement

This research was done without patient involvement.

\section{Design}

A 2-year prospective longitudinal design was followed in the present study. First, the heads of the schools were contacted by the research team, who explained the objectives and design of the study. In the next step, the physical education schoolteachers were contacted, since they would be in charge of educating schoolchildren after a previous BLS training.

Each school was randomly assigned to a group (figure 1), the control group (CG) or one of the two experimental groups. Initially, anthropometric data (weight and height) were registered, and the three groups were trained in BLS with the same methodology. Afterwards, the following two BLS assessments were carried out: a short-term evaluation within 1 week after the training (T1) and a long-term follow-up 2 years later (T2). Moreover, participants in the CG did not receive any additional intervention. One of the experimental groups, the standard group (SG), received one annual 50 min BLS retraining. The rolling-refresher group (RRG) received BLS rolling-refreshers every 4 months from T1 to T2 (adding up five sessions). Schools which did not attend all the trainings/rolling-refreshers or both assessments were excluded.

\section{Previous schoolteachers training}

Training was offered to all the teachers of the schools participating in the study; although, only physical education teachers participated in the training of the schoolchildren. The methodology and results regarding the training have already been discussed. Briefly, all the schoolteachers received the same instruction, as follows: 2 hours on BLS (out-of-hospital cardiac arrest (OOHCA), BLS sequence and compression-only CPR (CO-CPR), 40 min of theoretical contents and $80 \mathrm{~min}$ of practical skills (BLS sequence and CO-CPR). ${ }^{18}$

\section{Baseline whole sample training}

The initial training lasted $100 \mathrm{~min}$ (two 50 min physical education lessons in the same week). The first lecture 
comprised theoretical explanations about OOHCA, the importance of the immediate BLS and the different steps to assist an OOHCA following the BLS sequence. The second session consisted not only of reinforcing theoretical knowledge but also (and mainly) of practical skills. A rotational system of three groups was established. Each group trained on different skills at the same time. Every $15 \mathrm{~min}$, all groups rotated clockwise. The skills of each rotational group were (1) BLS sequence over manikintorso; (2) CO-CPR over basic manikin-torso; and (3) CO-CPR over Q-CPR manikin torso (with visual and auditory feedback). The manikin:pupil ratio was 1:5, and the schoolteacher:pupil ratio was 1:20-30, depending on the number of schoolchildren per classroom and those children who did not participate in the CPR lesson.

The following week after training, the BLS proficiency of schoolchildren was assessed during physical education lessons in a designated area. The same assessment was also carried out at T1 and T2. Evaluation was individual by means of an OOHCA simulated scenario. These assessments were carried out by members of the research team, all of whom were BLS or advanced life support instructors. Schoolchildren were told that they had to imagine their physical education schoolteacher collapsing and falling to the ground. A manikin placed on the floor played the role of the collapsed schoolteacher, and they were required to act. Observational evaluation of procedural skills was carried out, categorising the following BLS sequence variables as correctly performed/not correctly performed according to the ERC 2015 recommendations ${ }^{19}$ : (1) checking safety; (2) checking response; (3) opening airway; (4) checking breathing; (5) alerting emergency medical services (EMS); (6) starting CPR. The categorisation 'Not correctly performed' included the following two possibilities: an incorrectly performed step or a step not performed. In addition, the performance after 2 min of CO-CPR was also assessed. In the case that the participant did not start CPR by their own, the evaluator took the role of EMS dispatcher and reminded him or her to perform CPR. In this case, although 2 min of CPR could be evaluated, the item 'starting CPR' of the BLS sequence was categorised as 'not correctly performed'. If 'alerting EMS' was not performed either, the evaluator simulated a person who alerted EMS and provided the information to perform CPR to the participant. 'Alerting EMS' and 'starting CPR' were classified as 'Not correctly performed' in this case. CPR quality was measured and recorded with a Laerdal Resusci Anne QCPR (50-60 mm for depth compression and 100-120/com min for rate).

\section{Retraining and rolling-refreshers}

While CG only received the initial training, SG was retrained 1 year later, and RRG was involved in rollingrefreshers every 4 months. The retraining of SG was similar to the second lesson of the initial training. It was mainly practical, with a $50 \mathrm{~min}$ split in $5 \mathrm{~min}$ of theoretical overview of the BLS sequence and 45 min of practical skills retraining with the same rotational system as the first training.

According to the 'just-in-case' and 'just-in-place' education approaches, ${ }^{15}$ 5-min rolling-refreshers were implemented in the RRG. These very brief refreshers were carried out at physical education lessons. In groups of five schoolchildren, physical education schoolteachers refreshed children's memory in terms of BLS sequence. Afterwards, schoolchildren had to perform 2 min of CPR in groups of three children with one manikin for each one.

\section{Statistical analysis}

Variables are expressed as the mean (SD) or absolute frequencies (relative frequencies) as appropriate. Intergroup and intragroup analyses were carried out with BLS and CPR variables. All the BLS sequence variables were dichotomous. The $\chi^{2}$ test was used in the intergroup analysis to compare the three groups. To analyse differences between tests (intragroup: T1 vs T2), the McNemar test was used. Measured repeated ANOVA (MANOVA) was used to analyse CPR variables with the following two factors included in the model: intergroup (CG vs SG vs RRG) and intragroup (T1 vs T2) factors. Previously, ANCOVA was carried out for each CPR variable to determine and control possible covariate effects. In this sense, anthropometric variables were included as covariates in the model, since previous studies have shown correlations between anthropometry and CPR quality. ${ }^{8}$ In the MANOVA model, anthropometric variables with $\mathrm{p}<0.05$ reached in the ANCOVA analysis were included as covariates. The Pearson coefficient was used to analyse the linear correlation between anthropometric and CPR variables.

All the registered data were transferred to SPSS software (IBM Corp, V.23.0.0.0) for analysis. A significance level of $\mathrm{p}<0.05$ was considered in all analyses.

\section{RESULTS}

The final sample comprised 472 schoolchildren, since 186 were excluded due to not attending both training and/or evaluations and not signing informed consent or because of logistical problems in data collection. The distribution of participants was as follows: $C G, n=146$; SG, $\mathrm{n}=124$; RRG, $\mathrm{n}=202$.

Anthropometric variables were registered (weight, height and body mass index (BMI)) in both tests. No differences in any variable were found between groups at any time. However, as expected, due to the normal children growing pattern, significant changes were observed comparing T1 with T2 for the three variables in the three groups $(\mathrm{p}<0.001$ in all cases).

Figure 2 shows the proportion of participants performing each step of the BLS sequence in the intragroup analysis. At T2, there was a drastic decrease in CG participants in all steps of the BLS sequence except to start CPR $(p<0.05)$. Regarding SG, significantly fewer 

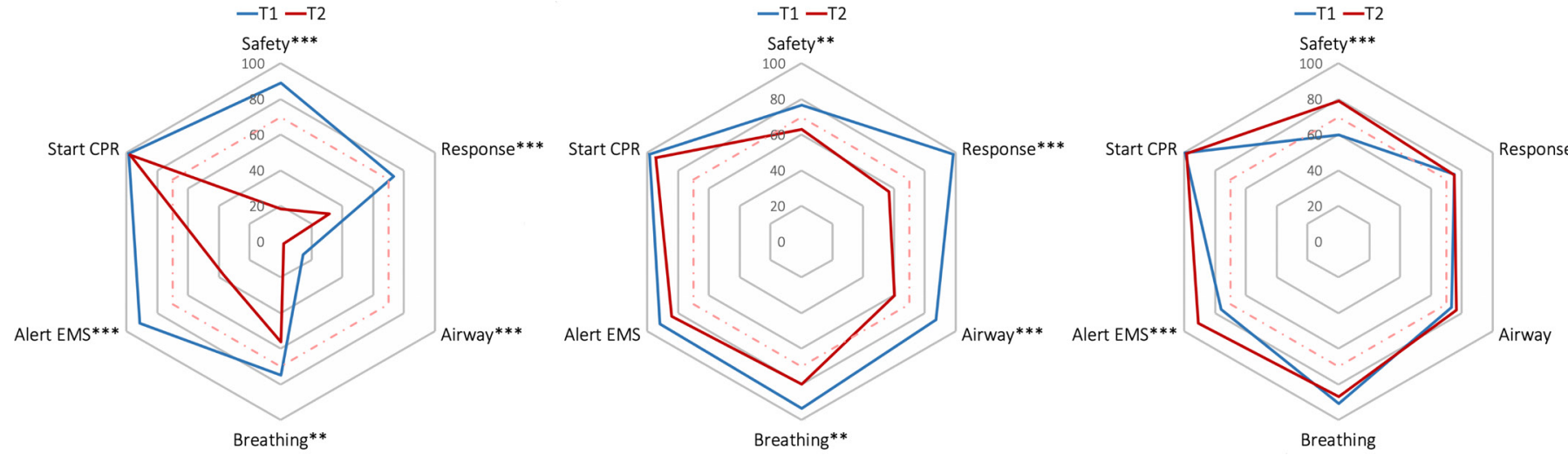

Figure 2 Intragroup analysis of BLS sequence. From left to right, CG-SG-RRG: ${ }^{*} \mathrm{p}<0.05,{ }^{* *} \mathrm{p}<0.001,{ }^{* \star *} \mathrm{p}<0.001$. BLS, basic life support; CG, control group; RRG, rolling-refresher group; SG, standard group.

participants were able to check safety, check response, open airway and breathing when T1 was compared with T2 $(p<0.05)$. Finally, the proportion of RRG participants able to perform any step of the sequence was similar at $\mathrm{T} 1$ and $\mathrm{T} 2$.

On the other hand, in the T2 intergroup analysis, except for the step 'starting CPR', the percentage of CG children who were able to perform any of the other BLS steps was significantly lower than that of SG and RRG children $(\mathrm{p}<0.05)$. When compared with SG children, RRG children reached a significantly higher proficiency in checking safety, checking response, opening airway and alerting EMS (figure 3). The only group in which each step of the BLS sequence was performed by at least $70 \%$ of participants was RRG.

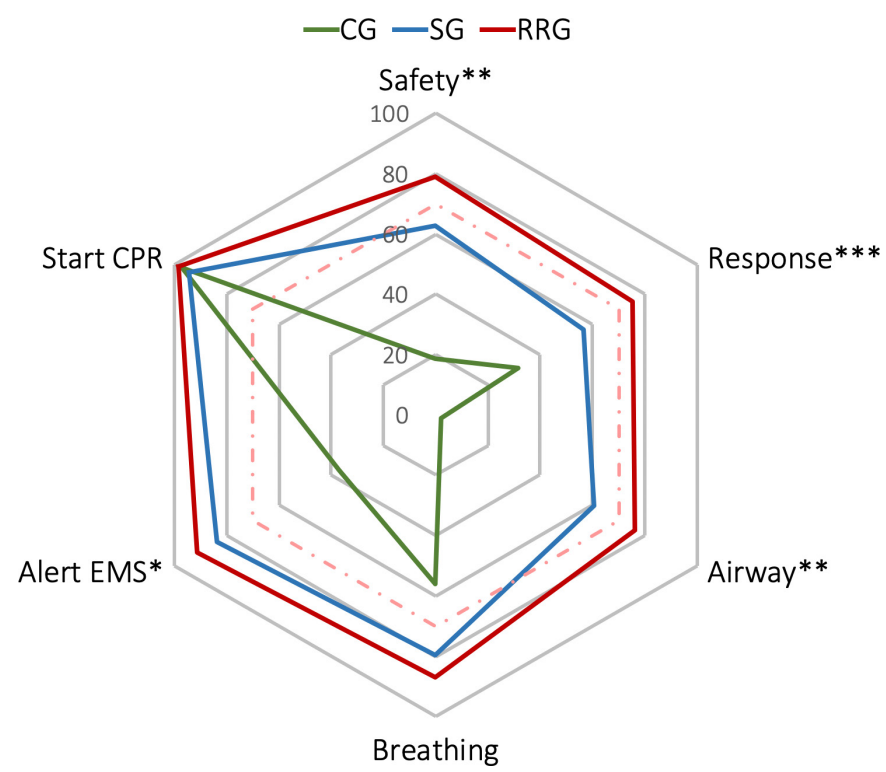

Figure 3 Intergroup analysis of BLS sequence in T2. Significant differences were found in all comparisons ( $p<0.001)$ CG vs $S G$ and CG vs RRG except for 'start CPR'. Asterisks show differences between SG and RRG: ${ }^{*} p<0.05,{ }^{* *} p<0.01,{ }^{* *} p<0.001$. BLS, basic life support; CPR, cardiopulmonary resuscitation; CG, control group; EMS, emergency medical services; RRG, rolling-refresher group; SG, standard group.
In a more stringent analysis of the BLS sequence, participants who correctly performed all the steps of the protocol were registered. Nobody in CG completed the entire BLS sequence in T2. In contrast, $28(22.6 \%)$ participants in the SG and $88(43.6 \%)$ in the RRG received it ( $p<0.001$ for all comparisons). These differences were significant between the $\mathrm{CG}$ and both experimental groups, as well as between the SG and RRG $(p<0.001$ in all cases).

Table 1 shows the data related to the quality of chest compressions (Q-CC), with intergroup and intragroup analysis adjusted by weight, height and BMI. In T2, the RRG reached higher figures for mean depth, correct CC by depth and Q-CC than the CG and SG $(\mathrm{p}<0.001)$. SG and RRG also obtained higher percentages of correct hand positions than CG $(\mathrm{p}<0.001)$.

Pearson correlation coefficients calculated between anthropometric variables and mean depth in T2 obtained a significant linear correlation independent of the group $(\mathrm{p}<0.001$ in all cases). However, Pearson coefficients were higher in CG than in SG and RRG and lower in the RRG than in the SG (figure 4). In this sense, a greater proportion of RRG participants (12.4\%) were able to reach at least a mean chest compression depth of $50 \mathrm{~mm}$ (CG: 6.2\%; SG: 8.1\%) (figure 4).

\section{DISCUSSION}

The present 2-year prospective longitudinal study aimed to compare the effect of annual retraining with very brief 4-month rolling-refreshers on the BLS skills of previously trained schoolchildren. We observed that schoolchildren involved in rolling-refreshers achieved better proficiency in both assessed skills, BLS sequence and Q-CC, than those not retrained at all or annually retrained peers. In terms of BLS sequence, higher proportions of RRG children were able to correctly perform the protocol steps. On the other hand, they also reached higher-quality CPR, especially in variables related to depth, although the absolute Q-CC was suboptimal.

The learning capacity of schoolchildren to perform BLS in simulated $d^{5-9} 1214$ and real conditions ${ }^{20}$ has already 
Table 1 Intragroup and intergroup analysis of CPR variables

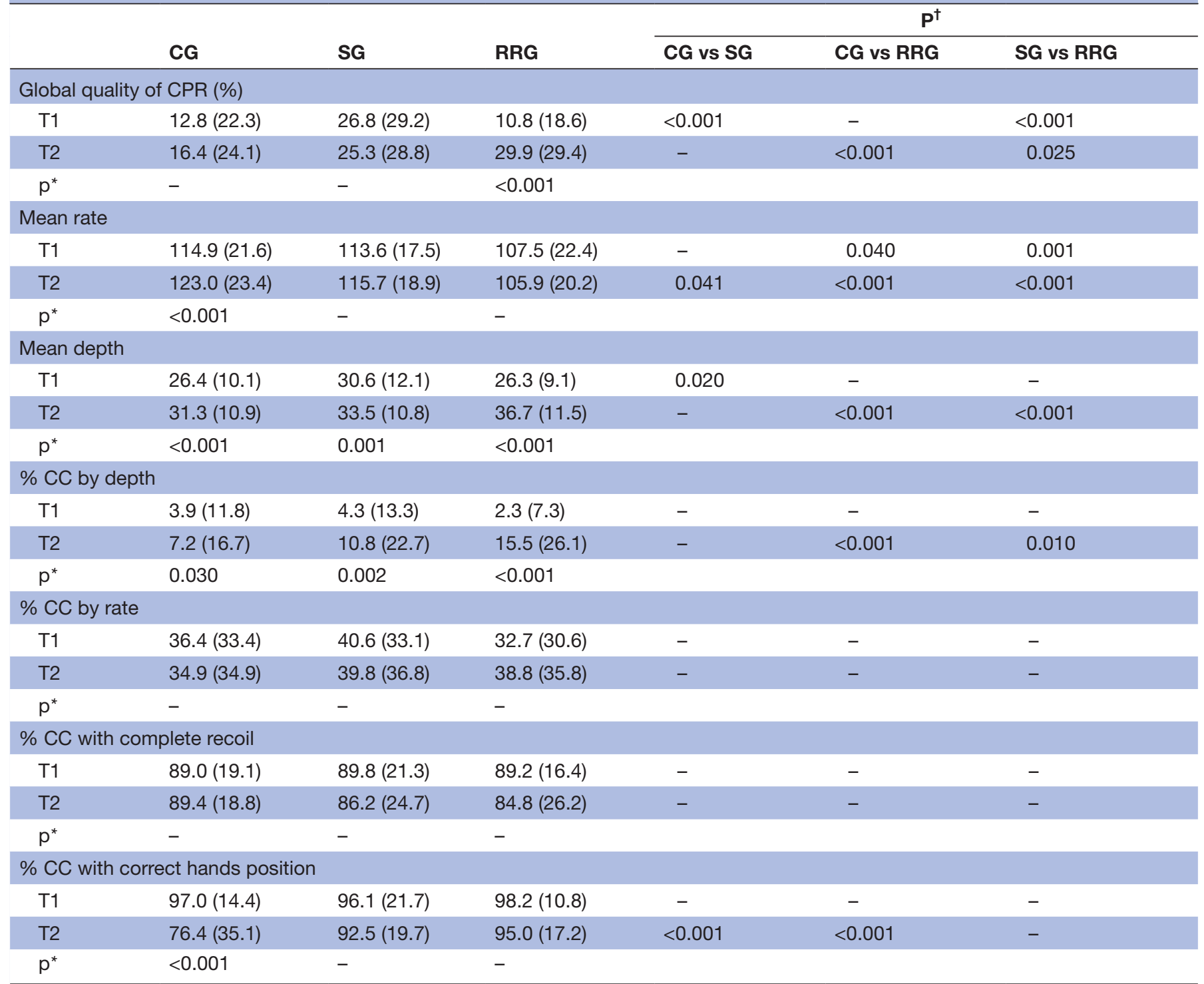

Continuous variables expressed as mean (SD).

*Intragroup analysis.

†Intergroup analysis.

CC, chest compression; CG, control group; CPR, cardiopulmonary resuscitation; RRG, rolling-refresher group; SG, standard group with retraining.

been demonstrated. In fact, some authors suggested that schoolchildren might learn BLS better and maintain knowledge over more time than adults. ${ }^{21}$ These are some of the reasons that BLS is strongly recommended to be taught in schools. ${ }^{2-4101114}$ In this sense, different investigations to develop evidence base around how BLS should be best taught in schools are needed. ${ }^{2}$

However, little is known about how often schoolchildren should train to maintain BLS knowledge/skills, and currently, 2 hours of training annually from the age of 12 years is recommended. ${ }^{11}$ As the year period may be too long for skills retention and perhaps it could not be easy to integrate 2-hour sessions into current school curricula, we tested two relatively innovative, feasible and practice-oriented strategies: brief annual retraining and very brief every 4 months of rolling-refreshers.

Regarding BLS sequence, a greater proportion of children in both experimental groups were able to perform the steps than CG 2 years after the first training, and at least $70 \%$ of the participants of RRG were able to perform each step correctly, reaching better proficiency than those schoolchildren of SG. Thus, 5 min rolling-refreshers were more effective than annual 50 min retraining in retaining how to correctly perform the BLS sequence. It must be considered that the BLS sequence requires acquiring much theoretical knowledge (to remember all the steps) and skills (to perform the steps correctly), which might be a reason why shorter refreshers shorter refreshers run 


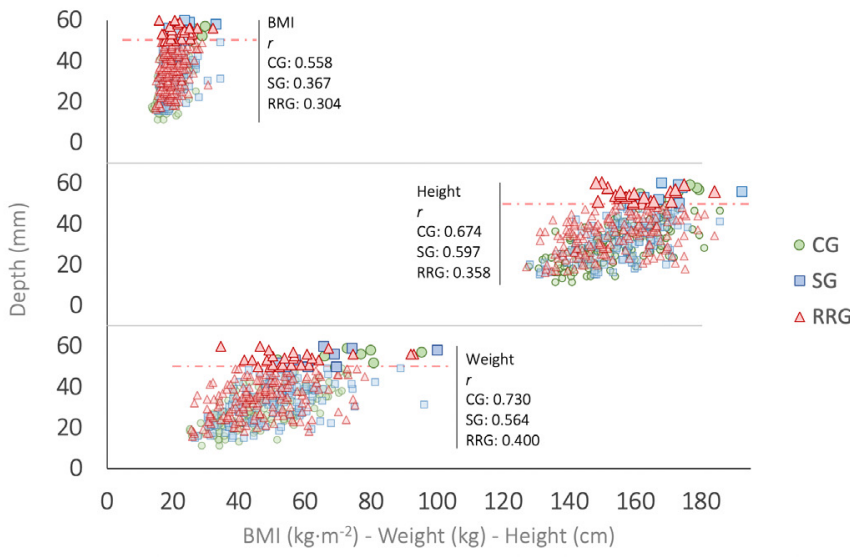

Figure 4 Relationships between anthropometric variables (X-axis: BMI, weight and height) and chest compression depth (Y-axis) with associated Pearson correlation coefficients. BMI, body mass index; CG, control group; RRG, rolling-refresher group; SG, standard group.

more frequently were effective in maintaining BLS skills over time.

In studies with healthcare staff and adult laypeople, rolling-refreshers strategies have been very useful to maintain CPR psychomotor skill competence. ${ }^{15} 16$ 22-24 and to remember the steps of the BLS sequence. ${ }^{17}$ In our study, rolling-refreshers allowed schoolchildren to reach higher-quality CPR, wherein the percentage of correct chest compression with adequate depth made the difference. Although the absolute CC depth was suboptimal considering hypothetical adult victims, at T2, participants from the three groups were able to compress deeper than in the baseline test. This sounds reasonable taking into account that the participants were schoolchildren who were in a growing stage of development, and their weight and height increased between both tests. Positive associations between anthropometric variables and the capacity to compress deeper were also shown not only in schoolchildren ${ }^{8}$ but also in adults. ${ }^{25}$ However, after adjusting the analysis by weight, height and BMI, our results showed that RRG compressed deeper than the other two groups, revealing that 4-month rolling-refreshers had a relevant impact in this sense. In any case, none of the three groups were able to reach the minimum chest compression depth $(50 \mathrm{~mm})$.

Skills retention is a main problem of life support training for both laypeople and healthcare professionals $^{22}$; thus, the frequency of rolling-refreshers is also a debated topic. Monthly refreshers were shown to be more effective than other training frequencies, such as every 3, 6 and 12 months in healthcare samples. ${ }^{72} 2627$ Nevertheless, the retention of BLS skills might depend on the specific content (theoretical/practice) and the age of the student. In the case of schoolchildren, we considered that in the current stage of BLS training at schools, such very frequent sessions might not be acceptable by schoolteachers, and we proposed every 4 months of retraining as an option easily embeddable into schools' schedule (one refresher taught in each of the three trimesters of academic year).

However, how can rolling-refreshers be implemented in school curricula schedules? Time devoted and logistics matter. In our study, although each schoolchildren only received hands-on 5 min training at each refresher session, schoolteachers had to spend a 50 min lesson, since they only had three manikins. Therefore, it might not be considered efficient in terms of time use to spend a whole lesson for only 5 min of training. In this regard, a recent publication suggested that low-cost handmade manikins might be adequate to teach and learn BLS skills at school. ${ }^{28}$ This option might be a cheap and feasible choice at any place that would also be advantageous in terms of time utilisation, since each child might have their own manikin. This would allow us to answer one question that emerged from our results regarding whether an increase in time of practice to 10 or $15 \mathrm{~min}$ would have a relevant impact on performance. Finally, another barrier to implementing BLS training at schools (at baseline, retraining and/or rolling-refreshers) is the training and engagement of schoolteachers. To solve this potential problem, in the present study, schoolteachers were previously taught in BLS; if BLS training was mandatory in universities, ${ }^{34}$ it would be easier to teach this content to schoolchildren.

\section{CONCLUSIONS}

In 8-to-12-year-old schoolchildren, although annual 50 min retraining sessions help to maintain BLS performance, 4-month very brief rolling-refreshers were shown to be even more effective. Thus, we recommend implementing baseline BLS training at schools, with subsequently brief rolling-refreshers.

\section{Author affiliations}

${ }^{1}$ Simulation and Intensive Care Unit of Santiago (SICRUS), Health Research Institute of Santiago, University Hospital of Santiago de Compostela- CHUS, Santiago

Compostela, Spain

${ }^{2}$ Faculty of Education Sciences, Universidade de Santiago de Compostela, Spain, Santiago Compostela, Spain

${ }^{3}$ CLINURSID Research Group, Psychiatry, Radiology, Public Health, Nursing and Medicine Department, University of Santiago de Compostela, Santiago de Compostela, Spain

${ }^{4}$ Faculty of Nursing, Universidade de Santiago de Compostela, Santiago Compostela, Spain

${ }^{5}$ REMOSS Research Group, Faculty of Education and Sport Sciences, University of Vigo, Vigo, Spain

${ }^{6}$ Faculty of Education and Sport Sciences, Universidad de Vigo, Vigo, Spain ${ }^{7}$ Pediatric, Health area of Santiago de Compostela-Barbanza, Santiago de Compostela, Spain

${ }^{8}$ Pediatric Intensive Care Unit, University Hospital of Santiago de Compostela-CHUS, Santiago de Compostela, Spain

Twitter Cristian Abelairas-Gómez @AG_Cristian_and Santiago Martinez-Isasi @C-1229-2017

Acknowledgements We would like to thank everyone who made possible this research, especially schoolteachers and schoolchildren.

Contributors CA-G, SM-I, RB-F and AR-N conceived the study. CA-G, SM-I, RB-F, CV-C, LSS and AR-N designed the study protocol, with input from the rest of the 
authors. The data were collected by AC-F, MP-L, FFM and MO-A. CA-G, SM-I and AC-F analysed the data. CA-G and AR-N drafted the manuscript and made revisions following critical review by all authors. SM-I is responsible for the overall content as guarantor. All authors approved the final version of the manuscript.

Funding Research funded by Instituto de Salud Carlos III - ISCIII- PI20/01355 Cofunded FEDER. AC-F (FPU19/02017) is recipient of a predoctoral fellowship by the Spanish Ministry of Science, Innovation and Universities.

Competing interests None declared.

Patient and public involvement Patients and/or the public were not involved in the design, or conduct, or reporting or dissemination plans of this research.

Patient consent for publication Consent obtained from parent(s)/guardian(s).

Ethics approval The study protocol has been approved by the Ethical Committee of the Faculty of Education and Sport Sciences - University of Vigo (Spain).

Provenance and peer review Not commissioned; externally peer reviewed.

Data availability statement Data are available upon reasonable request. Data available upon request to the author, smtzisasi@gmail.com.

Open access This is an open access article distributed in accordance with the Creative Commons Attribution Non Commercial (CC BY-NC 4.0) license, which permits others to distribute, remix, adapt, build upon this work non-commercially, and license their derivative works on different terms, provided the original work is properly cited, appropriate credit is given, any changes made indicated, and the use is non-commercial. See: http://creativecommons.org/licenses/by-nc/4.0/.

\section{ORCID iDs}

Cristian Abelairas-Gómez http://orcid.org/0000-0002-1056-7778

Santiago Martinez-Isasi http://orcid.org/0000-0001-8075-7668

Aida Carballo-Fazanes http://orcid.org/0000-0001-6615-9821

\section{REFERENCES}

1 Wissenberg M, Lippert FK, Folke F, et al. Association of national initiatives to improve cardiac arrest management with rates of bystander intervention and patient survival after out-of-hospital cardiac arrest. JAMA 2013;310:1377-84.

2 De Buck E, Van Remoortel H, Dieltjens T, et al. Evidence-based educational pathway for the integration of first aid training in school curricula. Resuscitation 2015;94:8-22.

3 Abelairas-Gómez C, Carballo-Fazanes A, Martínez-Isasi S. [Knowledge and attitudes on first aid and basic life support of Primary and Preschool teachers and parents]. An Pediatr 2020;92:268-76.

4 Abelairas-Gómez C, Schroeder DC, Carballo-Fazanes A, et al. Kids save lives in schools: cross-sectional survey of schoolteachers. Eur J Pediatr. In Press 2021;180:2213-21.

5 Martínez-Isasi S, Abelairas-Gómez C, Pichel-López M. [Learning to resuscitate at school. Study in 8-12 year-old schoolchildren]. An Pediatr 2020. [Epub ahead of print: 05 Nov 2020]. doi:10.1016/j. anpedi.2020.09.018

6 Meissner TM, Kloppe C, Hanefeld C. Basic life support skills of high school students before and after cardiopulmonary resuscitation training: a longitudinal investigation. Scand J Trauma Resusc Emerg Med 2012;20:31.

7 Banfai B, Pek E, Pandur A, et al. 'The year of first aid': effectiveness of a 3-day first aid programme for 7-14-year-old primary school children. Emerg Med J 2017;34:526-32.

8 Abelairas-Gómez C, Rodríguez-Núñez A, Casillas-Cabana M, et al. Schoolchildren as life savers: at what age do they become strong enough? Resuscitation 2014;85:814-9.

9 Jorge-Soto C, Abelairas-Gómez C, Barcala-Furelos R, et al. [Learning to use semiautomatic external defibrillators through audiovisual materials for schoolchildren]. Emergencias 2016;28:103-8.
10 Böttiger BW, Lockey A, Georgiou M, et al. Kids save lives: ERC position statement on schoolteachers' education and qualification in resuscitation. Resuscitation 2020;151:87-90.

11 Böttiger BW, Bossaert LL, Castrén M, et al. Kids Save Lives - ERC position statement on school children education in CPR.: "Hands that help - Training children is training for life". Resuscitation 2016;105:A1-3.

12 Otero-Agra M, Varela-Casal C, Castillo-Pereiro N, et al. [Can we train the chain of survival while playing? Validation of the tool «Rescube»]. An Pediatr. In Press 2021;94:213-22.

13 Borovnik Lesjak V, Šorgo A, Strnad M, Lesjak VB. Development, validation and assessment of the test on knowledge about basic life support and use of automated external defibrillator among schoolchildren. Scand J Trauma Resusc Emerg Med 2019;27:114.

14 Lukas R-P, Van Aken H, Mölhoff T, et al. Kids save lives: a six-year longitudinal study of schoolchildren learning cardiopulmonary resuscitation: who should do the teaching and will the effects last? Resuscitation 2016;101:35-40.

15 Niles D, Sutton RM, Donoghue A, et al. "Rolling Refreshers": a novel approach to maintain CPR psychomotor skill competence. Resuscitation 2009;80:909-12.

16 Niles DE, Nishisaki A, Sutton RM, et al. Improved Retention of Chest Compression Psychomotor Skills With Brief "Rolling Refresher" Training. Simul Healthc 2017;12:213-9.

17 González-Salvado V, Abelairas-Gómez C, Peña-Gil C, et al. Basic life support training into cardiac rehabilitation programs: a chance to give back. A community intervention controlled manikin study. Resuscitation 2018;127:14-20.

18 Pichel López M, Martínez-Isasi S, Barcala-Furelos R, et al. Un primer Paso en La enseñanza del soporte vital básico en LAS escuelas: La formación de Los profesores. Anales de Pediatría 2018;89:265-71.

19 Perkins GD, Handley AJ, Koster RW, et al. European resuscitation Council guidelines for resuscitation 2015: section 2. adult basic life support and automated external defibrillation. Resuscitation 2015;95:81-99.

20 Kurosaki H, Takada K, Yamashita A, et al. Patient outcomes of school-age, out-of-hospital cardiac arrest in Japan: a nationwide study of schoolchildren as witnesses. Acute Med Surg 2020;7:607.

21 Baldi E, Bertaia D, Contri E. School children learn BLS better and in less time than adults. Resuscitation 2015;88:e15-16.

22 Anderson R, Sebaldt A, Lin Y, et al. Optimal training frequency for acquisition and retention of high-quality CPR skills: a randomized trial. Resuscitation 2019;135:153-61.

23 Nishiyama C, Iwami T, Murakami Y, et al. Effectiveness of simplified 15-min refresher BLS training program: a randomized controlled trial. Resuscitation 2015;90:56-60.

24 Gonzï Lez-Salvado V, Abelairas-Gï Mez C, Peï A-Gil C, et al. A community intervention study on patients' resuscitation and defibrillation quality after embedded training in a cardiac rehabilitation program. Health Educ Res 2019;34:289-99.

25 Hong DY, Park SO, Lee KR, et al. A different rescuer changing strategy between 30:2 cardiopulmonary resuscitation and handsonly cardiopulmonary resuscitation that considers rescuer factors: a randomised cross-over simulation study with a time-dependent analysis. Resuscitation 2012;83:353-9.

26 So KY, Ko HF, Tsui CSY, et al. Brief compression-only cardiopulmonary resuscitation and automated external defibrillator course for secondary school students: a multischool feasibility study. BMJ Open 2020;10:e040469.

27 Chamdawala H, Meltzer JA, Shankar V, et al. Cardiopulmonary resuscitation skill training and retention in teens (CPR start): a randomized control trial in high school students. Resusc Plus 2021:5:100079.

28 Nakagawa NK, Oliveira KMG, Lockey A, et al. Effectiveness of the 40-Minute Handmade manikin program to teach Hands-on cardiopulmonary resuscitation at school communities. Am J Cardiol 2021;139:126-30. 